This article has been scanned by iThenticat No plagiarism detected

Volume 3, Issue 5, October 2021

p. $225-233$

\title{
SUSTAINBLE DEVELOPMENT AND THE EDUCATION TO COMBAT EPIDEMIC IN "L'ANNÉE DU LION" OF DEON MEYER
}

http://dx.doi.org/10.47832/2757-5403.5-3.20

\section{Racha Mohamed Hassan EL-ENANY ${ }^{1}$}

\begin{abstract}
:
Several years ago, before the emergence of the Corona virus that continues to sweep the world. A south African writer Deon Meyer predicts the coming of a virus that will kill $95 \%$ of the world's population.

This virus is a mixture of a human virus and a bat virus. It is almost the same thing we are experiencing right now.

Our work is based on a study of the story " L'Année Du Lion".

We focus on the friend ship son father. The education of gaining freedom and courage in the challenge of disease, defending the rights of others for the birth of a multinational society. Our research is divided into two parts.

The first part will be about the concept of education in this story and the second part about sustainable development that analyse Deon Meyer.
\end{abstract}

Key words: Sustainble Development, Education, Epidemic.

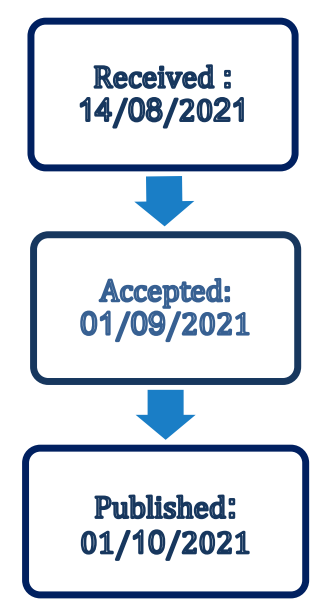

\footnotetext{
${ }^{1}$ Dr. , 6 October University , Mısır, rachaelenany77@gmail.com , https://orcid.org/0000-0003-1232-6414
}

Copyright (C) Published by IJHER Journal, www.ijherjournal.com Rimar Academy, Fatih, Istanbul, 34093 Turkey

All rights reserved 


\title{
LE DÉVELOPPEMENT DURABLE ET L'ÉDUCATION À LA LUTTE CONTRE L'ÉPIDÉMIE DANS " L'ANNÉE DU LION " CHEZ DEON MEYER
}

\section{Racha Mohamed Hassan E1 Enany}

\begin{abstract}
Résumé
Depuis quelques années, avant l'apparition de la pandémie du Corona Virus qui continue à ravager le monde entier, Deon Meyer, un écrivain d'Afrique du Sud, prévoit dans son roman "L'Année du Lion" l'arrivée d'un virus qui provoquera l'anéantissement de 95\% de la population du monde. Ce virus se forme de la fusion d'un virus humain et d'un virus de chauve souris, presque les mêmes évènements vecus ces jours-ci.

Notre travail se fondera sur l'étude de ce roman, "L'Année du Lion". On s'appuie sur la relation amicale entre un père et son fils, l'éducation à concevoir la liberté, le courage dans la lutte contre les maladies, la défense pour amener les droits d'autrui et la créativité pour la naissance d'une nouvelle communauté multiraciale.

Nous nous interesserons à former notre recherche de deux parties:La première partie sera consacrée à la notion de l'éducation dans ce roman et la deuxième partie présentera des axes du développement durable traités par Meyer tout au long de son écriture.

Mots- clés: Développement Durable, Éducation, Épidémie.
\end{abstract}

\section{Introduction:}

Depuis des années, plusieurs épidémies frappent le monde entier. Autrefois, c'était la peste, la Cholera, la variole, la grippe espagnole, la grippe porcine H1N1 et H2N2.

Avec toutes ces pandémies, Deon Meyer, l'écrivain de l'oeuvre "L'année Du Lion", prévoit l'arrivée d'un nouveau virus qui détruira le monde, le corona Virus. Ainsi, peut-on voir dans ce roman, la fiction avec beaucoup de signes et de leçons.

Notre travail se formera de deux parties. La première partie tourne autour de la relation entre le père et son fils. Le rôle de l'éducation dans l'affrontement des problèmes environnementaux, la confrontation des crises avec courage et force.

La deuxième partie traitera des axes du développement durable, discutés d'une manière indirecte tout au long de l'oeuvre, surtout avec la fondation de la communauté "Amanzi", fondée par le père avec l'intention de résoudre tous les problèmes dans une nouvelle cité vertueuse.

Comment est ce que le père et son fils, les héros de cette oeuvre, arriveront à surmonter la crise du "virus Corona "? Comment Willem storm, le père, arrivera à construire une nouvelle communauté ? C'est ce que on essayera d'élucider dans le travail présent.

Nous nous reposerons sur les théories et les idées des théoriciens spécialisés

en psychologie et en pédagogie, tel que John Dewey, qui s'oriente dans sa théorie moderne vers le naturalisme; "les activités de l'enfant étaient toujours au centre de ses préoccupations et qu'il n'y a pas de différences entre experience de vie et d'apprentissage" (Dewey, 1916)

l'homme doit verifier mettre des hypothèses pour arriver aux conclusions "Que signifie la démocratie, si ce n'est que l'individu doit avoir son mot à dire dans la détermination des conditions et des buts de son propre travail" (Dewey, 1916)

Willem storm observera toutes les menaces environementales qui attaquent son pays durant la crise du Corona et cherche à trouver les solutions en commençant à reconstruire 
une communauté vierge, là où il établit l'ordre, la justice, la paix et résoudra le problème de la famine qui engloutit son pays.

\section{La première partie:}

\section{Le Rôle De L'éducation Dans L'affrontement Du Danger}

Dés la première partie du roman, on entend la voix du fils, Nico Storm, qui est en train d'utiliser des pronoms personnels sujets entre la première personne et la deuxième personne du singulier comme s'il veut partager son histoire avec son lecteur. "c'est l'histoire de ma vie. Et l'histoire de ta vie et de ton monde, tu verras" (Meyer, 2016) ${ }^{1}$

\section{Education à travers l'encouragement et la tranquilisation:}

Nico storm essaie de sauver ses souvenirs du relachement, il commence à relater ses mémoires à l'âge de 47 ans. Il retourne à l'âge de 13 ans, durant les années de la catastrophe. A chaque paragraphe, on remarque de la sagesse dans l'éducation du père à son fils.

"j'ai demandé à père s'il pensait que nous allions tomber malade, il m'a répondu que les gens allaient trouver une solution, les médecins, les gouvernements et les instituts scientifiques, je ne devais pas m'inquiéter. L'humanité a toujours un plan de secours." (Meyer, 2016, p. 40)²

Dans le premier chapitre, le père est mordu par quelques chiens, il sauve son fils de tout danger sans lui faire peur ce qui est remarqué d'après quelques mots comme "Il se tient immobile pour protéger son enfant.... Le désespoir dans sa

voix est comme une décharge et balaye ma peur"3 (Meyer, 2016, p. 14).

La partie précédente dénote le dévouement du père, l'adaptation et la conversion dans l'éducation selon Jhon Dewey, malgré tout le mal qu'il est en train de sentir, il apprend à son fils la stabilité émotionnelle.

Lorsque Nicolas demande à son père des questions à propos de la chauve souris, le père explique doucement en tranquilisant son fils avec tendresse "Il m'ébouriffe les cheveux" (Meyer, 2016, p. 14)

De même, le père après être attaqué, il serre son fils contre ses bras pour le calmer.

\section{Education à avoir de la responsabilité et l'apprentissage à travers les experiences:}

Malgré toutes les mauvaises circonstances subies par la famille Storm, le père aide son fils à avoir un rôle et à lui partager la responsabilité. C'est ce que le théoricien, pédagogue, Dewey appelle l'apprentissage expérientiel. l'enfant doit faire l'expérience et des activités libres pour apprendre à subir les problèmes.

"Moi, je m'occupe du long tuyau d'arrosage. Il est raccordé à la pompe électrique et je dois mettre l'embout dans le réservoir du camion et attendre" (Meyer, 2016, p. 14)

Sur le point de mourir et entre les griffes des chiens, le père encourage son fils pour tuer ces animaux.

"Prends le pistolet, Nico. Tire!

Ce n'est pas un ordre mais une supplication anxieuse, comme si

mon père voyait déjà la mort et les conséquences pour son fils: seul,

en rade, condamné"

Willem storm apprend à son fils d'être courageux, de surmonter la crise pour ne pas vivre seul après la mort de son père.

D'abord le coup était non maitrisé puis petit à petit l'enfant s'encourage et

"tire, encore et encore" (Meyer, 2016, p. 16) ${ }^{2}$

2-CHOME (Etienne),La Méthode Critère pour mieux gérer nos conflits,UCL, p.60, 2009 
Le père apprend à son fils les premiers secours et à bien agir surtout durant les mauvaises circonstances, Nicolas essaie de nettoyer les blessures de son père en se dévouant pour le sauver.

Même avec la souffrance du père, il continue à encourager son fils, à le tranquiliser. Ce qui apprend à Nico à se fortifier pour affronter tous ces problèmes.

"Le but de l'éducation est de permettre à chaque individu d'entrer en pleine possession de ces facultés personnelles" (Dewey, 1916)

Willem Storm éduque son fils à compter sur soi dans toutes les conditions de la vie et même durant les mauvaises circonstances.

Et malgré l'insistance du père à transmettre au fils la force et le courage, il arrive à lui transmettre l'amour qui se traduit par la sensibilité consciente du fils

"Je sens le sang et la sueur sur mon père, me colle à lui".

Nicolas est un enfant sensible et avec toutes les mauvaises circonstances qui l'entourent, il devient hypersensible et bienveillant.

Il essaie de comprendre les mots de son père et de décoder des énigmes qui ne sont que des directives et des hypothèses prévoyés par le père mais transmis indirectement à l'enfant dans des buts éducatifs.

"Et ses yeux, ternes, dans le vague. Brusquement, je comprends ses silences et tout le reste. La peur m'envahit

et je fonds en larmes." (Meyer, 2016, p. 17)

On peut toucher à chaque phrase la sensibilité de l'enfant qui essaie malgré tout de s'efforcer pour subir avec son père les conséquences du virus.

\section{Education pour agir avec sagesse}

Storm continue à encourager son fils et lui fournir de l'ambition. Il veut lui apprendre d'essayer de réparer "tant qu'on peut " (Meyer, 2016, p. 20). tout ce qui est mauvais et de travailler jusqu'au dernier moment. Le père apprend à Nicolat à se reconcilier, même durant les moments dures, pour agir avec sagesse.

C'est la notion de compétence, selon Dewey, "agir en situation, avec efficacité et avec sagesse". ${ }^{2}$ (Dewey, 1916, pp. -https://journals.openedition.org/edso/6308)

Dans la plupart des parties de ce roman, avec la catastrophe, le manque de sécurité, le père est en train de donner à son fils des leçons éducatives.

"Je sais que tu as peur, Nico, mais je vais être malade un ou deux jours"3 (Meyer, 2016, p. 24)

Le fils se sent responsable et plus âgé. Il essaie de reposer son père et de lui montrer qu'il est capable de l'aider à surmonter la catastrophe. "je réchauffe

la soupe sur la cuisinière à gaz. Velouté de poulet de Baxters"4 (Meyer, 2016, p. 24).

Selon Dewey, l'éducation doit favoriser la croissance des enfants à travers "leurs pouvoirs" 5(https://www.erudit.org/fr/revues/phro/2016-v5-n2-phro02850/1038137ar/) c'est à dire, apprendre à l'enfant à tirer expérience des situations.

L'enfant apprend même à se suffir des minimes des choses. Il oublie ses besoins et ses demandes avec le cauchemar du virus, dans une société de consommation anéantie.

\section{Education à suivre les conseils et les directives}

Willem apprend à son fils à suivre ses directives, entendre ses renseignements et de continuer à les appliquer. Malgré la maladie de son père, comme Willem lui a dit "On ne fait confiance à personne" 6(Meyer, 2016, p. 27) Nico refuse d'appeler les gens qu'il trouve hors de la maison pour les sauver.

Toujours en continuant à suivre les directives du père, l'enfant garde le calme et la discretion pour que les personnes qu'il est en train de voir à travers les rideaux, ne violent pas leur maison. 
Même au péril de sa vie, en tenant le pistolet en main, il suit les principes de son père et refuse de tuer l'homme qui le terrorise avec sa voix près de la porte.

De même, il utilise son intelligence pour s'échapper des voleurs qui entourent leur maison, il se calme et s'immobilise en reprenant les idées de son père "Il m'a appris que les sons portent bien plus loin qu'on ne pense" 7 (Meyer, 2016, p. p 30)

L'enfant fait même attention que l'odeur du café peut attirer les autres vers eux, ce qui dénote la bienveillance du père et son insistance à soutenir son fils avec tous les moyens malgré qu'ils sont prises avec la catastrophe.

Le père aide son fils pour arriver à une appréhension minutieuse de tous les côtés du problème vécu pour pouvoir le surmonter.

\section{Education à avoir un esprit scientifique:}

Willem éduque son fils à bien comprendre les faits de mettre des hypothèses et des conclusions pour bien résoudre les problèmes et créer pour renouveler.

"L'évaluation est pour nous le processus qui consiste à recueillir un ensemble d'informations pertinentes, valides et fiables, et d'examiner le degré d'adéquation entre cet ensemble d'informations et un ensemble de critères choisis adéquatement en vue de fonder une prise de precision" (Guingouain, p. 25)

Le père, dans "l'Année Du Lion", prépare son fils pour l'aider à la formation d'une nouvelle communauté construite sur des bases scientifiques.

"Il ne me parle jamais comme à un enfant. Il ne filtre pratiquement jamais les informations, c'est à moi de lui dire si je ne comprends pas."

Willem traite son fils comme un jeune homme et non pas un adolescent. Il prévoit dans l'image de son fils, un héros qui l'aidera à réaliser son rêve de la fondation d'un nouveau monde sans virus, ni mal, ni humiliation.

Nico est éduqué à respecter les sciences, les normes de l'environnement et l'usage de la technologie pour le bien du monde.

\section{Education à être courageux et sauver les autres}

Malgré la maladie de willem storm, le père, qui est attaqué par le Covid encourage son fils pour l'aider à sauver la femme battue et torturée par les hommes qui assiègent leur maison. C'est toujours l'apprentissage par l'action, selon Dewey, l'expérience continue. C'est l'éducation qui forme les hommes.

Dewey demande que l'éducation soit basée sur l'aide mutual entre les gens "le développement du sens social implique communication, entraide, coopération, collaboration et intérêt mutuel." (Meyer, 2016) https://www.memoireonline.com/05/08/1095/m_methodes-actives-systeme-educatif-

Storm apprend à son fils d'aider les autres jusqu'au dernier moment.

Storm forme son fils à respecter les valeurs et les principes même en agissant avec des ennemis. Le père, Willem, refuse d'attaquer l'homme qui essaie de le tuer. Le fils répète que son père ne peut pas tirer sur les hommes.

"La paix sur la terre dépend de notre capacité à protéger notre environnement vivant."

Tout au long du roman "L'Année Du Lion", le père est en train de fournir à son fils des idées, (Sylvie, 2009, p. 81) des informations et parfois de faire allusion aux différentes notions du développement durable.

\section{Deuxième Partie}

\section{Le Développement Durable Dans "L'Année Du Lion"}

Depuis les premières pages de "L'Année Du Lion", L'auteur nous laisse voir plusieurs notions du développement durable. 
"Le developpement est un processus global, économique, social, culturel et politique, qui vise à améliorer sans cesse le bien-être de l'ensemble de la population et de tous les individus, sur la base de leur participation active libre et significative au développement et au partage équitable des bienfaits qui en découlent" (Sylvie, 2009, p. 8)

Le père, dans "L'année du lion", cherche à rétablir la terre démolie par les virus, former une nouvelle communauté, Amanzi, qui signifie "l'eau". Storm voudra irriguer une nouvelle terre par la communicabilité, la coopération, l'honneur et le respect des droits de l'homme.

"On va repartir de zéro ici. Fonder une communauté avec un sens

moral, des principes, une considération mutuelle. Il faut que tout soit

bien dès le début." (Meyer, 2016, p. 40)

le père commence au début du roman par expliquer à son fils le sens de certains mots qui avaient leurs orientations parmi les piliers de l'environnement, de la sociologie et de l'économie.

Il a le but de "fonder une communauté " (Meyer, 2016, p. 40) selon les normes de la cité vertueuse. Recommencer une nouvelle vie pure sans épidémie ni frustration.

"Le monde, maintenant... Nous allons l'arranger, Nico, le raccommoder.

Toi et moi." (Meyer, 2016, p. 55)

\section{L'élecricité, L'écologie et le développement durable dans "L'Année Du Lion"}

Pour la reconstruction d'une nouvelle communauté, l'auteur est en train de montrer l'état sur laquelle se trouve le pays, après l'influence du virus. La disparition de la plupart des formes d'évolution.

"Dans la lueur d'une lampe à gaz" (Meyer, 2016, p. 18),"un petit réchaud à gaz" (Meyer, 2016, p. 20)

L'électricité est le moyen d'évolution de n'importe quel endroit et joue un rôle écologique important. L'auteur insiste à projeter la lumière sur toutes les traces de la pandémie qui seront les éléments essentiels étudiés par Willem pour la construction de sa nouvelle colonie Amanzi.

Storm parle de "l'énergie hydroélectrique" (Meyer, 2016, p. 39) qui peut être produit par les habitants de la nouvelle communauté.C'est un héros qui rêve de la modification en tous les domaines

Storm rêve d'une ville impeccable, productrice et développée. il cherche l'énergie renouvelable "Augmenter la capacité de production sur la base de ressources locales, principalement le gaz naturel et l'hydroélectricité". (Revue, juin 2021)

Willem Storm trouve dans le village qu'il visite avec son fils le bon exemple pour réaliser son projet. Il essaie de l'étudier et de comprendre son état avant et après l'épidémie pour essayer de résoudre les problèmes.

"un pont, un barrage, un vrombissement. gauche, le lac, parfaitement immobile, une vaste étendue d'eau scintillante. droite, une rivière dans une gorge profonde, voilée derrière la brume de l'eau qui bouillonne sur les écluses (..)

Je pense qu'ils ont laissé les écluses ouvertes" Storm suit tous les défauts parmi lesquels des problèmes écologiques et par conséquence économiques.

"La crise du Covid -19 a déferlé comme un tsunami sur les économies africaines" (Revue, juin 2021, p. 170)

Tous les problèmes sanitaires affectent la société et causent un désastre économique.

En décrivant Vanderkloof, Storm pense aussi à l'agriculture, comment faire revire son pays.

Storm cherche à sauver son pays et le reste de l'humanité de l'extinction. Toutes les ressources commencent à être épuisées. Tout est en peril.

"Toutes les maisons vides" (Meyer, 2016, p. 22) ceci dénote l'augmentation du taux de la mortalité durant ces années de fièvre. 


\section{La Protection de l'environnement et le développement durable dans "L'Année du Lion":}

Le père qui prépare pour la construction d' une nouvelle communauté cherche à protéger l'environnement. Avec espoir et ambition, il insiste à affronter le problème.

Il choisit la terre Vanderkloof pour trouver l'eau nécessaire pour l'agriculture, le retour de l'élevage et la sécurité alimentaire.Il mentionne qu'il y a des rivières Il cite que même le climat de cet endroit est "clément" (Meyer, 2016, p. 39)

"Un jour nous aurons à nouveau du lait frais" (Meyer, 2016, p. 35).

Il veut construire l'asile sous lequel les gens peuvent se refugier et trouver l'abri, la sécurité et le Bonheur.

L'écrivain de" L'Année Du Lion " envisage le problème du réchauffement climatique.

L'enfant Nico est tout au long du roman en train de parler de la chaleur, de l'étouffement qui aggravent le problème.

Le père Willem storm cherche l'endroit où il pourra trouver l'eau, comme ressource de vie. il essaye d'éviter dans sa nouvelle communauté, la possibilité des conflits:

"conflits pour l'eau, pour la nourriture, pour fuir les zones atteintes par une chaleur excessive" (Fred, 2019, p. 201)

\section{La sociologie, le besoin de sécurité et le developpement durable dans "L'Année du Lion":}

A force de subir du mal durant les années de la pandémie, Storm cherche tous les moyens par lesquels il veut renaitre les valeurs humaines perdues par l'humiliation, le viol et 1'harcèlement:

"Nous nous souvenons le mieux des traumatismes. La peur, la perte, l'humiliation" (Meyer, 2016, p. 40)

Willem Storm explique à son fils que l'homme doit recevoir ses droits, reprendre sa sécurité et recommencer sa vie après les années de fièvre.

La référence de Storm à la pyramide de Maslow est un moyen de transmettre à son fils l'idée des besoins psychologiques et physiologiques des personnes.

"la pyramide des besoins selon Abraham Maslow" (Meyer, 2016, p. 39).c'est la pyramides qui montre les besoins de l'homme, la sécurité, la confiance en soi, le respect, la motivation, l'estime...etc.

Willem expose à son fils les effets negatifs de la pandémie et les moyens de les concevoir avec les solutions possibles.

Durkheim fondateur de la sociologie comme science empirique, souligne le fait que "les notions de la vie sociale sont générées du fait des délimitations qui sont établies entre ce qui est propre et ce qui est impropre." (Frédéric, 2014, p. 39)

Durkheim explique que les relations sociales ne sont pas limitées, la communication doit être continue et l'aide mutuel empêche l'amour personnel.

Le père, fondateur d'Amanzi, essai avant tout de d'établir la bonne relation entre les habitants de sa nouvelle communauté.

"cohésion sociale, l'harmonie

entre

êtres

humains" https://journals.openedition.org/developpementdurable/1133

Le père Storm encourage son fils au bénévolat et à pouvoir aider les autres volontairement. Malgré tous les problèmes, Storm est en péril de sa vie mais insiste à prêter attention à la femme kidnapée et fait tout ce qui lui est possible pour lui rendre la sécurité et la vie.

"Père se lève en titubant. Il se tient le cou. Du sang filtre à travers

ses doigts. Du sang qui paraît noir" (Meyer, 2016, p. 46).

La femme était sur le point de mourir violée, battue et harcelée dans un monde qui est devenu barbare, qui donne survie au plus fort.

"Nous l'entendons pleurer doucement" (Meyer, 2016, p. 52) 
Storm est tout le temps en train de défendre la femme, les droits de l'homme et l'environnement.

Le fondateur d'Amanzi vise à ce que le peuple ne soit pas "obligé de subir une cohabitation percue comme dégradante, humiliante, avec une population elle même dégradée, méprisée, dépréciée." (Pierre, 1993)

\section{Conclusion}

Enfin, on peut déduire après la lecture de ce roman et la présentation de notre recherche que l'écrivain, à force de vivre des années successives avec l'apparition de plusieurs virus, il arrive à prévoir la pandémie coronavirus qui infecte le monde entier.

L'auteur de "L'Année du Lion" décrit la catastrophe avec minutie comme s'il est en train de photographier nos jours-ci.

Il cite des expressions utilisés depuis le commencement de la Pandémie Corona

Tel que: "une infection", "bactérien", "la fièvre", "une angine", "un rhume" (Meyer, 2016, p. 21)

Même on peut entendre les héros du roman, en racontant comment le virus est transmis à l'homme à travers un autre malade "séropositif" (Meyer, 2016, p. 25) et à travers les animaux, surtout les chauves souris attaquées par la maladie.

En traitant dans notre recherche des axes de l'éducation et du développement durable, on explore des sujets qui menacent l'humanité, qui doivent être des thèmes de recherches et de discussions.

L'écrivain arrive à mentionner à travers les différents évènements de ce roman postapocalyptique l'importance du travail mutuel pour reconstruire un monde sain, juste équitable et civilisé.

Deon Meyer, anticipe les évènements de la misère du monde, vécue durant nos jours comme s'il veut attirer l'attention sur le futur de l'humanité et le rôle des sciences dans l'affrontement des problèmes environnementaux.

Meyer fait un tour pré-Covid (Revue, juin 2021, p. 170) des impacts de ce virus sur la population, il commence par l'explication de la transmission du virus de l'animal à l'homme puis il expose le nombre de mortalité qui augmente à chaque instant, le réchauffement climatique qui peut ravager la vie sur terre, l'agriculture qui se dégrade.

L'écrivain de "l'Année du Lion" prévoit le manque des ressources qui doivent être renouvelables, le manque de la production et de la consommation affectant le commerce mondial.

Avec la construction d'Amanzi qui signifie eau, c'est à dire la vie; Meyer cherche un futur prospère, là ou tous les soucis seront transformés en sécurité et en paix.

Entre tous ces problèmes Meyer n'oublie pas de traiter le sujet de la psychologie des hommes. Il cherche tous les moyens par lesquels les pays peuvent préserver à l'être son humanité, ses droits et son respect.

Lorsque Storm sauve la dame des hommes qui la menacent, il fait flèche pour traiter le sujet de l'émancipation de la femme, l'un des axes indéniables du développement durable.

Pour conclure, on peut dire que ce travail est un message au monde entier pour se réveiller, se méfier et prendre des mesures fermes pour résoudre les problèmes.

\section{Bibliographie}

Dewey, John. (1916)

Fred, Vargas. (2019). L'Humanité en péril.Flammarion

Frédéric, Keck. (2014). Les sentinelles des pandémies. Essais.

Guingouain, G. (n.d.). Psychologie sociale et Evaluation.

https://www.erudit.org/fr/revues/phro/2016-v5-n2-phro02850/1038137ar/. (n.d.(.

Meyer, Deon. (2016). L'année du lion. France: seuil.. 
Pierre, Bourdieu. (1993). la Misère du Monde. France: Seuil.

Revue. (juin 2021).Jeune afrique. Une Histoire Africaine.

Sylvie, Brunel. (2009). Le Développement Durable. France: PUF.

Todd, E. (n.d.). Où sommes-nous? seuil. 